Addendum

\title{
Addendum: Yang, Z., et al. Multi-Toxic Endpoints of the Foodborne Mycotoxins in Nematode Caenorhabditis elegans. Toxins (Basel), 2015, 7(12), 5224-5235
}

\author{
Zhendong Yang ${ }^{1,2}$, Kathy S. Xue ${ }^{2}$, Xiulan Sun ${ }^{1}$, Lili Tang ${ }^{1,2, *}$ and Jia-Sheng Wang ${ }^{2}$ \\ 1 Synergetic Innovation Center of Food Safety and Nutrition, School of Food Science, Jiangnan University, \\ Wuxi, Jiangsu 214122, China; zdyang777@163.com (Z.Y.); sxlzzz@jiangnan.edu.cn (X.S.) \\ 2 Department of Environmental Health Science, University of Georgia, Athens, GA 30602, USA; \\ ksxue@uga.edu (K.S.X.); jswang@uga.edu (J.-S.W.) \\ * Correspondence: ltang@uga.edu; Tel.: +1-706-542-9765; Fax: +1-706-542-7472 \\ Academic Editor: Vernon L. Tesh \\ Received: 27 April 2016; Accepted: 29 April 2016; Published: 5 May 2016
}

The authors wish to add the first affiliation address "Synergetic Innovation Center of Food Safety and Nutrition, School of Food Science, Jiangnan University" to the correspondence author, Lili Tang's affiliations on the first page of their paper published in Toxins [1]. The authors apologize for any inconvenience this may cause.

For any questions, please contact the editorial office of the journal or support@mdpi.com.

\section{Reference}

1. Yang, Z.; Xue, K.S.; Sun, X.; Tang, L.; Wang, J.S. Multi-toxic endpoints of the foodborne mycotoxins in nematode caenorhabditis elegans. Toxins (Basel) 2015, 7, 5224-5235. [CrossRef] [PubMed]

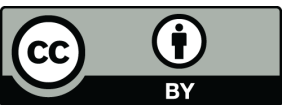

(C) 2016 by the authors; licensee MDPI, Basel, Switzerland. This article is an open access article distributed under the terms and conditions of the Creative Commons Attribution (CC-BY) license (http:/ / creativecommons.org/licenses/by/4.0/). 